\title{
Estimation of radiation cancer risk in CT-KUB
}

\begin{abstract}
The increased demand for computed tomography (CT) in radiological scanning examinations raises the question of a potential health impact from the associated radiation exposures. Focusing on CT kidney-ureter-bladder (CT-KUB) procedures, this work was aimed at determining organ equivalent dose using a commercial CT dose calculator and providing an estimate of cancer risks. The study, which included 64 patients (32 males and 32 females, mean age 55.5 years and age range 30-80 years), involved use of a calibrated CT scanner (Siemens-Somatom Emotion 16-slice). The CT exposures parameter including tube potential, pitch factor, tube current, volume CT dose index $\left(\mathrm{CTDI}_{\mathrm{vol}}\right)$ and dose-length product (DLP) were recorded and analyzed using CT-EXPO (Version 2.3.1, Germany). Patient organ doses, including for stomach, liver, colon, bladder, red bone marrow, prostate and ovaries were calculated and converted into cancer risks using age- and sex-specific data published in the Biological Effects of Ionizing Radiation (BEIR) VII report. With a median value scan range of $36.1 \mathrm{~cm}$, the $\mathrm{CTDI}_{\mathrm{vol}}$, DLP, and effective dose were found to be $10.7 \mathrm{mGy}, 390.3 \mathrm{mGy} \mathrm{cm}$ and $6.2 \mathrm{mSv}$, respectively. The mean cancer risks for males and females were estimated to be respectively 25 and 46 out of 100,000 procedures with effective doses between $4.2 \mathrm{mSv}$ and $10.1 \mathrm{mSv}$. Given the increased cancer risks from current CT-KUB procedures compared to conventional examinations, we propose that the low dose protocols for unenhanced CT procedures be taken into consideration before establishing imaging protocols for CT-KUB.
\end{abstract}

Keyword: CT-KUB; Patient effective dose; Organ equivalent dose and cancer risks 